\title{
Magnetic force signal of vortex creation in type-II superconducting thin films
}

\author{
J.C. Wei ${ }^{a}$, L. Horng ${ }^{b, *}$, T.J. Yang ${ }^{a}$ \\ a Department of Electro-Physics, National Chiao-Tung Unilersity, Hsinchu, Taiwan, ROC

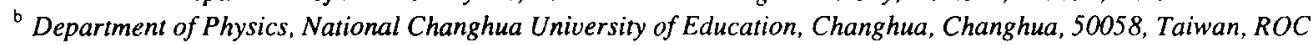

Received 27 August 1996; revised 24 March 1997; accepted 27 March 1997

\begin{abstract}
The possibility of the formation of a vortex in thin film due to the field of magnetic dipole moment when a point dipole is vertically approaching the surface of a thin film is discussed. Calculations are performed for certain discrete values of the critical position of the point dipole for creating the vortex in the thin film and the equilibrium positions of the vortex, both of which depend on the strength of magnetic dipole moment. The creation of a new single vortex in the thin film causes an abrupt change in the vertical force. (C) 1997 Elsevier Science B.V.
\end{abstract}

\section{Introduction}

Magnetic force microscopy (MFM) has been applied to high- $T_{\mathrm{c}}$ superconductors, especially to investigate the flux line structures [1,2]. In particular, low-temperature is a powerful technique for accessing information on the stray field near the surface of superconductors. The mathematical modeling of the interaction of a stray magnetic field with a test magnet is a key to describing the magnetic force of MFM system. In this fascinating research area, the theories related to the interaction of magnetic fields with a magnetic probe tip for various geometry of superconductors have been established [3-8]. In a recent paper [7] we introduced a general theoretical approach to calculating the magnetic forces acting on

\footnotetext{
* Corresponding author. Tel.: + 886 (4) 7232105 ext. 3324 ; fax: + 886 (4) 721-1153; e-mail: plhorng@ncue4680.ncue.edu.tw.
}

a point dipole above an infinite superconducting thin film in the mixed state based on the London theory.

In this work we consider the possibility of the formation of vortices in a thin superconducting film only in the field of magnetic dipole moment, i.e., the creation is due to the effect of vortices which are induced by the magnetic field of the dipole on the scanning process. We investigate the critical positions of the point dipole for creating the vortex in the thin film and the equilibrium distances between vortices for a various number (including the first, second and third vortices) of vortices to be created in sequence in the thin film. We also calculate the change of magnetic force acting on the point dipole after the vortex creation.

Assuming that the superconducting film lies in the $x y$ plane with infinite area, and the point dipole tip is a given distance from the film boundary. The magnetic field of the dipole on the boundary of the film 
would be very small and negligible. The magnetic field near the upper pole and the lower pole have a stronger field, so we consider the appearance of a flux penetration in the film just below the dipole tip. From the energy point of view, a vortex can form in the film as the strength of field on the film surface increases, if the creation of a vortex is energetically favorable. This vortex penetrates perpendicular to the film surface, unlike the vortex penetration parallel to the surface of the semi-infinite sample for the Bean-Livingston case. One should note that the interaction of a vortex with its mirror image, just as has been done for Bean-Livingston surface barrier, should be removed. We can calculate the change of system energy after a vortex creation in the film.

Let us consider a magnetic point dipole with a moment $\boldsymbol{m}$ placed above an infinite superconducting film with a single vortex embedded in it. The energy of a vortex in the superconducting thin film is

$U_{\mathrm{v}}=\frac{\phi_{0}^{2}}{2 \pi \mu_{0} \Lambda} \ln \left(\frac{\Lambda}{\xi}\right)$.

Where $\phi_{0}$ is the flux quantum $h / 2 \mathrm{e}, \mu_{0}$ is the vacuum permeability, $\Lambda$ is the effective penetration depth, and $\xi$ is the coherence length. In the case of the single vortex in the superconducting film the interaction energy of the MFM system has been obtained $[7,8]$ and the resulting interaction energy is

$$
\begin{aligned}
U_{\mathrm{int}}(a)= & U_{\mathrm{p}-\mathrm{p}}(a)+U_{\mathrm{p}-\mathrm{v}}\left(a, r_{0}\right) \\
= & \frac{\mu_{0} m^{2}}{8 \pi} \int_{0}^{\infty} \mathrm{d} k \frac{k^{2}}{k \Lambda+1} \mathrm{e}^{-k a} \\
& -\frac{m \phi_{0}}{2 \pi} \int_{0}^{\infty} \mathrm{d} k \frac{k}{k \Lambda+1} J_{0}\left(k r_{0}\right) \mathrm{e}^{-k a},
\end{aligned}
$$

where $a$ is the separation between the dipole and the thin film, and $r_{0}$ is the lateral distance between the dipole and the vortex in the plane of thin film, which have the polar coordinates $(r, \theta)$. The first term and the second term on the right-hand side of Eq. (2) represent the self-interaction energy [3,5-8] $\left(U_{\mathrm{p}-\mathrm{p}}\right)$ caused by the screening currents (Meissner effect) and the interaction energy $\left(U_{\mathrm{p}-\mathrm{v}}\right)$ between the dipole and the vortex, respectively. Then the magnetic forces acting on the point dipole are

$$
\begin{aligned}
F_{z}= & \frac{\mu_{0} m^{2}}{4 \pi} \int_{0}^{\infty} \mathrm{d} k \frac{k^{3}}{K \Lambda+1} \mathrm{e}^{-2 k a} \\
& -\frac{m \phi_{0}}{2 \pi} \int_{0}^{\infty} \mathrm{d} k \frac{k^{2}}{K \Lambda+1} J_{0}\left(k r_{0}\right) \mathrm{e}^{-k a} \\
= & F_{1}(a)+F_{2}\left(a, r_{0}\right), \\
F_{r 0}= & -\frac{m \phi_{0}}{2 \pi} \int_{0}^{\infty} \mathrm{d} k \frac{k^{2}}{k \Lambda+1} J_{1}\left(k r_{0}\right) \mathrm{e}^{-k a},
\end{aligned}
$$

where $F_{z}$ is the vertical force and $F_{r 0}$ is the later force. $F_{1}$ and $F_{2}$ are the forces acting on the point dipole in vertical $(z)$ direction respectively caused by the screening currents and the vortex. The interaction force between the dipole and the vortex is attractive, and the interaction force between the dipole and the screening currents is repulsive.

\section{Conditions of vortex creation}

To simplify the analysis of vortex creation into the superconducting thin film, we assume a vortex is formed perpendicularly through the thin film one at a time when the dipole is vertically approaching the film surface along the $r=0$ axis in $-z$ direction.

We consider the direction of the dipole moment normal to the plane of a type-II thin film of thickness $d(d \ll \lambda, \lambda$ is the London penetration depth), and the dipole tip to be placed at a distance $a$ above the surface of film. Below, we discuss the conditions of the creation of the first, second and third vortex in the thin film, respectively.

\subsection{Creation of the first vortex}

When a point dipole tip is lowered toward the surface of a superconducting film, persistent currents in the film are established which produce a magnetic field opposing that of the point dipole. The free energy of the system in the Meissner state is the self-interaction energy in Eq. (2) plus the self-energy of the point dipole. For expressing the free energy, we skipped the self-energy of the point dipole in the following calculation, because it is a constant term which diverges as the radius of the point dipole approaches to zero. 
Further lowering the height of the dipole may create a vortex in the film because of an increase in magnetic field. Now we shall consider the creation of the first vortex in the thin film, if the point dipole is lowered to a position $a_{1}$. In order to find the critical position $a_{1}$, we need to compare the free energy of in the absence of a vortex and the free energy of the appearance of the first vortex compared to none in thin film. The free energy of the system before, $U_{0}$, and after, $U_{1}$, the creation of the first vortex are obtained, respectively, as follows.

$$
\begin{aligned}
U_{0}\left(a_{1}\right)= & U_{\mathrm{p}-\mathrm{p}}\left(a_{1}\right) \\
= & \frac{\mu_{0} m^{2}}{8 \pi} \int_{0}^{\infty} \mathrm{d} k \frac{k^{2}}{k \Lambda+1} \mathrm{e}^{-2 k a_{1}}, \\
U\left(a_{1}\right)= & U_{\mathrm{p}-\mathrm{p}}\left(a_{1}\right)+U_{\mathrm{p}-\mathrm{v}}\left(a_{1}, 0\right)+U_{\mathrm{v}} \\
= & \frac{\mu_{0} m^{2}}{8 \pi} \int_{0}^{\infty} \mathrm{d} k \frac{k^{2}}{K \Lambda+1} \mathrm{e}^{-k a_{1}} \\
& -\frac{m \phi_{0}}{2 \pi} \int_{0}^{\infty} \mathrm{d} k \frac{k}{K \Lambda+1} \mathrm{e}^{-k a_{1}}+U_{\mathrm{v}} .
\end{aligned}
$$

Here we assume the first vortex is created just below the point dipole.

The critical position of the point dipole $a_{1}$ for creating the first vortex in thin film can be found by setting $U_{0}=U_{1}$. Then $a_{1}$ can be determined by the following equation,

$\left[\frac{\Lambda}{a_{1}}-\mathrm{e}^{a_{1} / \Lambda} \mathrm{E}_{1}\left(\frac{a_{1}}{\Lambda}\right)\right] \alpha=\ln \left(\frac{\Lambda}{\xi}\right)$,

where $\alpha=\mu_{0} m / \phi_{0} \Lambda$ reflects the normalized strength of the magnetic moment, and $\mathrm{E}_{1}\left(a_{1} / \Lambda\right)$ is an exponential integral. In Fig. 1, the critical position $a_{1}$ of the creation of the first vortex is plotted as a function of $\alpha$ for $\Lambda / \xi=10^{3}$. To get analytic expression of $a_{1}$, two extreme situations are considered, from Eq. (7) we obtain

$$
\begin{aligned}
& a_{1}=\sqrt{\frac{\alpha}{\ln \left(\frac{\Lambda}{\xi}\right)}}, \quad \text { for } \alpha \gg 1, \\
& a_{1}=\sqrt{\frac{\alpha \Lambda}{\ln \left(\frac{\Lambda}{\xi}\right)}}, \quad \text { for } \alpha \ll 1 .
\end{aligned}
$$

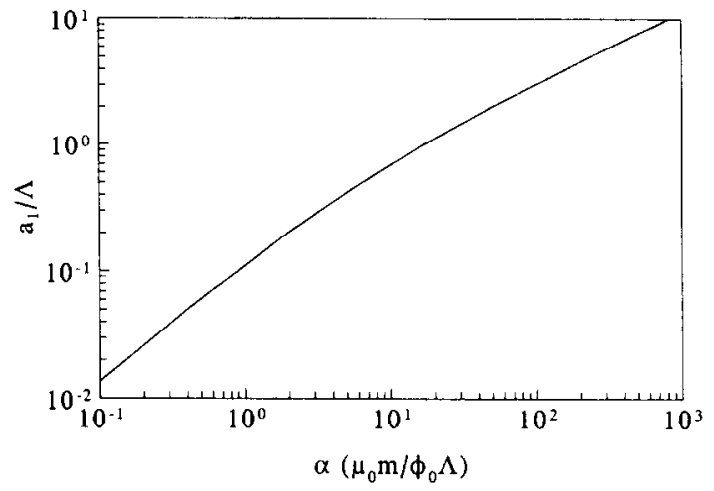

Fig. 1. The critical position $a_{1}$, which is the position of the first vortex creation in the thin film, as a function of $\alpha$ for the parameter $\Lambda / \xi=10^{3}$.

It is clear that $a_{1}$ depends on the strength of the magnetic moment. Both Eqs. (8) and (9) are reasonable by considering the problem in the appropriate cases. For $\alpha \gg 1$ case, for instance, the stronger the field a magnetic dipole moment has, the higher the position of the creation of the first vortex in the film is expected.

Let us find the change in magnetic force acting on the point dipole when the first vortex is created in the film. The forces acting on the dipole before, $F_{z}$, and after, $F_{z 1}$, the creation of the first vortex can be obtained, respectively, as follows

$F_{z}-F_{1}\left(a_{1}\right)$,

$F_{z 1}=F_{1}\left(a_{1}\right)+F_{2}\left(a_{1}, 0\right)$,

where $F_{z}$ and $F_{z 1}$ represent the vertical force on the point dipole with none and one quanta of the flux in thin film, respectively. Eq. (11) shows clearly how the vertical force changed as the first vortex appears in the film. The force $F_{1}$ caused by the screening currents is still the same after the vortex creation. An additional force $F_{2}$, caused by the vortex, is found after the vortex creation. The difference in the vertical force between the first vortex appears and no vortex exists in the film, $\left|\Delta F_{2}\right|$, is

$$
\begin{aligned}
\left|\Delta F_{z}\right|= & \left|F_{2}\left(a_{1}\right)\right| \\
= & \frac{m \phi_{0}}{2 \pi \Lambda^{3}}\left[-\left(\frac{\Lambda}{a_{1}}\right)+\left(\frac{\Lambda}{a_{1}}\right)^{2}\right. \\
& \left.+\mathrm{e}^{a_{1} / \Lambda} \mathrm{E}_{1}\left(\frac{a_{1}}{\Lambda}\right)\right] .
\end{aligned}
$$


Taking two limitations in Eq. (12), we have

$\left|\Delta F_{z}\right|=\frac{m \phi_{0}}{\pi a_{1}^{3}}\left(1-3 \frac{\Lambda}{a_{1}}\right)$, for $a_{1} \gg \Lambda$,

$\left|\Delta F_{z}\right|=\frac{m \phi_{0}}{2 \pi a_{1}^{2} \Lambda}, \quad$ for $a_{1} \ll \Lambda$.

\subsection{Creation of the second vortex}

When the point dipole continues to approach the surface of thin film, increasing in magnetic field, the possibility of creation of the second vortex appears. To perform the corresponding calculations we should know the interaction energy of two vortices. The interaction energy between two vortices in the case of infinitely thin superconducting film was obtained $[9,10]$ and is of the form

$U_{12}(r)=\frac{\phi_{0}^{2}}{2 \mu_{0} \Lambda}\left[H_{0}\left(\frac{r}{\Lambda}\right)-Y_{0}\left(\frac{r}{\Lambda}\right)\right]$,

where $r$ is the distance between two vortices, $H_{0}$ is the Struve function of order zero and $Y_{0}$ is the Bessel function of the second kind of order zero. If the point dipole is lowered down to a position $a_{2}$, the creation of the second vortex in thin film appears. The free energy before, $U_{1}$, and after, $U_{2}$, the creation of the second vortex, respectively, may be written as

$$
\begin{aligned}
U_{1}\left(a_{2}\right)= & U_{\mathrm{p}-\mathrm{p}}\left(a_{2}\right)+U_{\mathrm{p}-\mathrm{v}}\left(a_{2}, 0\right)+U_{\mathrm{v}}, \\
U_{2}\left(a_{2}\right)= & U_{\mathrm{p}-\mathrm{p}}\left(a_{2}\right)+2 U_{\mathrm{p}-\mathrm{v}}\left(a_{2}, r_{1}\right)+2 U_{\mathrm{v}} \\
& +U_{12}(r),
\end{aligned}
$$

where $r=2 r_{1}$ is the distance between two vortices and $r_{1}$ is the equilibrium position of the vortex from the origin (or equilibrium center). We assume that there exists a position of stable equilibrium for the vortex, the attractive force between the dipole and the vortex and the repulsive force between two vortices balanced each other. We do not consider these two vortices coinciding because they need more energy to form a two-quanta vortex. In the following we express the procedures to find $a_{2}$ and $r_{1}$.

The critical condition that the system free energy before and after the creation of the second vortex is equal for the system $U_{1}\left(a_{2}\right)=U_{2}\left(a_{2}\right)$; we have

$2 U_{\mathrm{p}-\mathrm{v}}\left(a_{2}, r_{1}\right)-U_{\mathrm{p} \cdot \mathrm{v}}\left(a_{2}, 0\right)+U_{\mathrm{v}}+U_{12}(r)=0$.

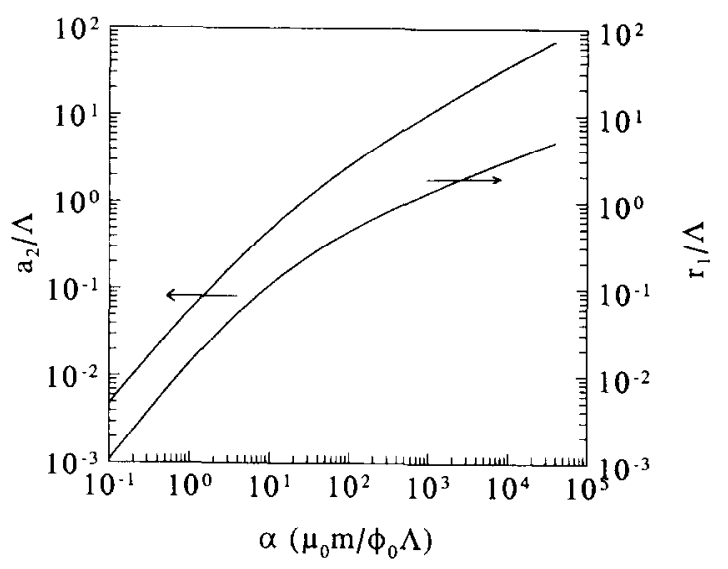

Fig. 2. The critical position $a_{2}$ of the creation of the second vortex and the equilibrium positions $r_{1}$ of the vortices as a function of $\alpha$ for the parameter $A / \xi=10^{3}$.

The condition for equilibrium of vortices is

$F_{r}=-\frac{\partial U_{2}\left(a_{2}\right)}{\partial r_{1}}=0$.

Applying these two conditions of Eqs. (18) and (19) to determine $a_{2}$ and $r_{1}$; we have

$$
\begin{aligned}
& \alpha \int_{0}^{\infty} \mathrm{d} t \frac{t \mathrm{e}^{-\left(a_{2} / \Lambda\right) t}}{t+1}\left[2 J_{0}\left(\frac{r_{1}}{\Lambda} t\right)-1\right] \\
& =\ln \left(\frac{\Lambda}{\xi}\right)+\pi\left[H_{0}\left(\frac{2 r_{1}}{\Lambda}\right)-Y_{0}\left(\frac{2 r_{1}}{\Lambda}\right)\right], \\
& \alpha \int_{0}^{\infty} \mathrm{d} t \frac{t \mathrm{e}^{-\left(a_{2} / \Lambda\right) t}}{t+1} J_{1}\left(\frac{r_{1}}{\Lambda} t\right) \\
& \quad=\pi\left[H_{1}\left(\frac{2 r_{1}}{\Lambda}\right)-Y_{1}\left(\frac{2 r_{1}}{\Lambda}\right)-\frac{2}{\pi}\right] .
\end{aligned}
$$

Fig. 2 shows the critical position $a_{2}$ and the equilibrium position $r_{1}$ versus $\alpha$ (the normalized strength of the magnetic moment). The height of the critical position $a_{2}$ is lower than that of the critical position $a_{1}$ for the samc reduced magnet moment. The change in vertical force acting on the point dipole due to the second vortex formation in the thin film can be written as

$$
\left|\Delta F_{z}\right|=\frac{m \phi_{0}}{2 \pi} \int_{0}^{\infty} \mathrm{d} k \frac{k^{2} \mathrm{e}^{-k a_{2}}}{k \Lambda+1}\left[2 J_{0}\left(k r_{1}-1\right] .\right.
$$

This integral is finite and convergent. In the case $r_{1} \ll \Lambda$, we can limit ourselves to a second-order 
calculation, i.e., the approximation $J_{0}\left(k r_{1}\right) \cong 1-$ $\left(\left\{k^{2} r_{1}^{2}\right) / 4\right.$, then we obtain

$$
\begin{aligned}
\left|\Delta F_{z}\right|= & \frac{m \phi_{0}}{2 \pi \Lambda^{3}}\left[-\left(\frac{\Lambda}{a_{2}}\right)+\left(\frac{\Lambda}{a_{2}}\right)^{2}+\mathrm{e}^{a_{2} / \Lambda} \mathrm{E}_{1}\left(\frac{a_{2}}{\Lambda}\right)\right] \\
& -\frac{m \phi_{0}}{4 \pi \Lambda^{3}}\left(\frac{r_{1}}{\Lambda}\right)^{2}\left[-\left(\frac{\Lambda}{a_{2}}\right)+\left(\frac{\Lambda}{a_{2}}\right)^{2}\right. \\
& \left.-2\left(\frac{\Lambda}{a_{2}}\right)^{3}+6\left(\frac{\Lambda}{a_{2}}\right)^{4}+\mathrm{e}^{a_{2} / \Lambda} \mathrm{E}_{1}\left(\frac{a_{2}}{\Lambda}\right)\right] .
\end{aligned}
$$

From the result of Fig. 2, we see that the values of $a_{2}$ are much smaller than the effective penetration depth $\Lambda$ for $r_{1} \ll \Lambda$. We use here the condition $a_{2} \ll \Lambda$; from Eq. (23) we obtain

$\left|\Delta F_{z}\right|=\frac{m \phi_{0}}{2 \pi \Lambda^{3}}\left(\frac{\Lambda}{a_{2}}\right)^{2}\left[1-3\left(\frac{r_{1}}{a_{2}}\right)^{2}\right]$.

For small $r_{1} / \Lambda(<0.1)$, the values of $r_{1} / a_{2}$ are about 0.21 to 0.26 for a range of $\alpha$ from 0.1 to 10 .

\subsection{Creation of the third vortex}

In the following, let us treat the problem of three vortices. For simplicity, we assume that these vortices to form an equilateral triangle. Following similar procedure, we have

$$
\begin{gathered}
\alpha \int_{0}^{\infty} \mathrm{d} t \frac{t \mathrm{e}^{-\left(a_{3} / A\right) t}}{t+1}\left[3 J_{0}\left(\frac{r_{3}}{\Lambda} t\right)-2 J_{0}\left(\frac{r_{2}}{\Lambda} t\right)-1\right] \\
=\ln \left(\frac{\Lambda}{\xi}\right)-\pi\left[H_{0}\left(\frac{2 r_{2}}{\Lambda}\right)-Y_{0}\left(\frac{2 r_{2}}{\Lambda}\right)\right] \\
\quad+3 \pi\left[H_{0}\left(\frac{\sqrt{3} r_{3}}{\Lambda}\right)-Y_{0}\left(\frac{\sqrt{3} r_{3}}{\Lambda}\right)\right] \\
\alpha \int_{0}^{\infty} \mathrm{d} t \frac{t^{2} \mathrm{e}^{-\left(a_{3} / \Lambda\right) t}}{t+1} J_{1}\left(\frac{r_{2}}{\Lambda} t\right) \\
=\pi\left[H_{1}\left(\frac{2 r_{2}}{\Lambda}\right)-Y_{1}\left(\frac{2 r_{2}}{\Lambda}\right)-\frac{2}{\pi}\right]
\end{gathered}
$$

$$
\begin{aligned}
& \alpha \int_{0}^{\infty} \mathrm{d} t \frac{t^{2} \mathrm{e}^{-\left(a_{3} / \Lambda\right) t}}{t+1} J_{1}\left(\frac{r_{3}}{\Lambda} t\right) \\
& =\sqrt{3} \pi\left[H_{1}\left(\frac{\sqrt{3} r_{3}}{\Lambda}\right)-Y_{1}\left(\frac{\sqrt{3} r_{3}}{\Lambda}\right)-\frac{2}{\pi}\right],
\end{aligned}
$$

where $a_{3}$ is the critical position of the point dipole for creating the third vortex in the film and $r_{2}$ and $r_{3}$ specify the equilibrium positions of the vortex from the origin for the system before and after the creation of the third vortex, respectively. Fig. 3 shows the critical position $a_{3}$ and the equilibrium position $r_{2}$ and $r_{3}$ versus $\alpha$ (the normalized strength of the magnetic moment). The critical position $a_{3}$ becomes closer to the surface of the film than those of $a_{2}$ and $a_{1}$. It also can be seen from Fig. 3 that the equilibrium position of the vortex (or the distance between vortices) becomes farther than the position it was after the creation of the third vortex. The change in vertical force acting on the dipole after the creation of the third vortex in the film is

$$
\begin{aligned}
\left|\Delta F_{z}\right|= & \frac{m \phi_{0}}{2 \pi} \int_{0}^{\infty} \mathrm{d} k \frac{k^{2} \mathrm{e}^{-k a_{1}}}{k \Lambda+1} \\
& \times\left[3 J_{0}\left(k r_{3}\right)-2 J_{0}\left(k r_{2}\right)\right] .
\end{aligned}
$$

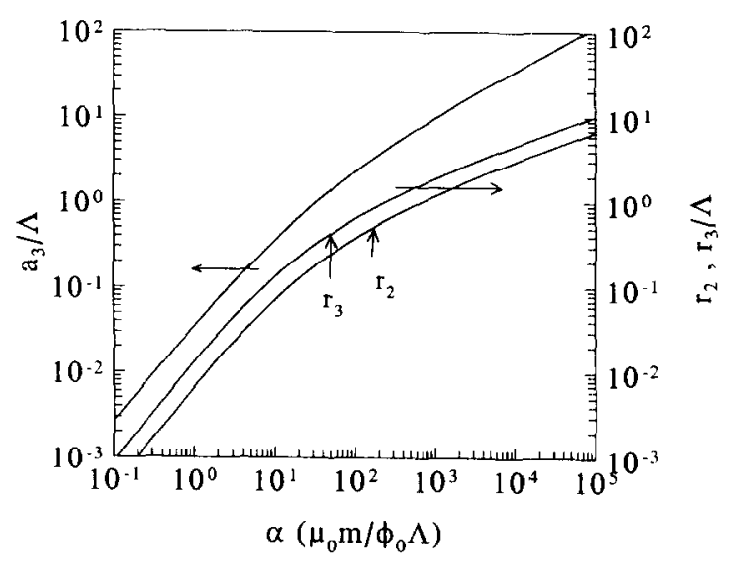

Fig. 3. The critical position $a_{3}$ of the creation of the third vortex and the equilibrium positions $r_{2}$ and $r_{3}$ as a function of $\alpha$ for the parameter $\Lambda / \xi=10^{3}$, where $r_{2}$ and $r_{3}$ specify the equilibrium positions of the vortex before and after the formation of the third vortex, respectively. 
Again in the $r_{2}, r_{3} \ll \Lambda$ limit, we have

$$
\begin{aligned}
\left|\Delta F_{z}\right|= & \frac{m \phi_{0}}{2 \pi \Lambda^{3}}\left[-\left(\frac{\Lambda}{a_{3}}\right)+\left(\frac{\Lambda}{a_{3}}\right)^{2}+\mathrm{e}^{a_{3} / \Lambda} \mathrm{E}_{1}\left(\frac{a_{3}}{\Lambda}\right)\right] \\
& -\frac{m \phi_{0}}{8 \pi}\left(\frac{3 r_{3}^{2}-2 r_{2}^{2}}{\Lambda^{5}}\right)\left[-\left(\frac{\Lambda}{a_{3}}\right)+\left(\frac{\Lambda}{a_{3}}\right)^{2}\right. \\
& \left.-2\left(\frac{\Lambda}{a_{3}}\right)^{3}+6\left(\frac{\Lambda}{a_{3}}\right)^{4}+\mathrm{e}^{a_{3} / \Lambda} \mathrm{E}_{1}\left(\frac{a_{3}}{\Lambda}\right)\right],
\end{aligned}
$$

and

$\left|\Delta F_{z}\right|=\frac{m \phi_{0}}{2 \pi \Lambda^{3}}\left(\frac{\Lambda}{a_{3}}\right)^{2}\left[1-\frac{3}{2}\left(\frac{3 r_{3}^{2}-2 r_{2}^{2}}{a_{3}^{2}}\right)\right]$,

for $a_{3} \ll \Lambda$.

The values of $r_{2} / a_{3}$ and $r_{3} / a_{3}$ are about 0.17 to 0.20 and 0.34 to 0.39 , respectively, for a range of $\alpha$ from 0.1 to 10 .

\section{Conclusions}

We have demonstrated that presence of the field of a point dipole tip may lead to vortex formation in thin superconducting films. The condition of the vortex creation and the change in magnetic force on the point dipole for various numbers of vortices, including the first, second and third vortices cases, in the absence of free from pinning center in the thin film have been examined. There is a change in vertical force which acts on the point dipole when a new single vortex is created in the film. The force changes discontinuously as the vortex is created in the film. In the free of pinning centers case, there is no lateral force signal owing to the symmetry of vortex geometry. The numerical results of the critical positions of the point dipole for creating the vortex and equilibrium positions of the vortex for various strengths of the magnetic moment can help us to realize the microscopic character of the MFM.

\section{Acknowledgements}

We thank Dr. J.L. Chen for helpful discussions and correspondence. This research was supported by the National Science Council of the Republic of China under Grant No. NSC 86-2112-M-009-007-PH and Grant No. NSC 85-2112-M-018-007.

\section{Keferences}

[1] P. Rice, J. Moreland, IEEE Trans. Magn. 27 (6) (1991); A. Moser et al., J. Vac. Sci. Technol. B 12 (1994) 1586.

[2] H.J. Hug, A. Moser, I. Parashikov, B. Stiefel, O. Fritz, H.-J. Güntherodt, H. Thomas, Physica C 235-240 (1994) 2695.

[3] Z.J. Yang, Jpn. J. Appl. Phys. 31 (1992) L477; 31 (1992) L938; J. Supercond. 5 (1992) 259; Physica C 234 (1994) 263.

[4] A. Wadas, O. Fritz, H.J. Hug, H.-J. Güntherodt, Z. Phys. B 88 (1992) 317.

[5] J.H. Xu, J.H. Miller Jr., C.S. Ting, Phys. Rev. B 51 (1995) 424.

[6] M.W. Coffey, Phys. Rev. B52 (1995) 1187; B52 (1995) R9851; M.W. Coffey, E.T. Phipps, Phys. Rev. B53 (1996) 389.

[7] J.C. Wei, J.L. Chen, L. Horng, T.J. Yang, Phys. Rev. B 54 (1996) 15429.

[8] J.C. Wei, J.L. Chen, L. Homg, T.J. Yang, Physica C 267 (1996) 345.

[9] J. Pearl, Ph.D. thesis, Polytechnic Institute of Brooklyn, 1965.

[10] J. Pearl, in: J.G. Daunt, D.O. Edward, F.J. Milford, M. Yagub (Eds.), Low Temperature Physics LT9, Plenum, New York, 1965, p. 566. 